\title{
Crushing analysis and multi-objective crashworthiness optimization of GFRP honeycomb-filled energy absorption devices
}

\author{
J. Paz*, J. Díaz*, L. Romera*, M. Costas* \\ Structural Mechanics group, School of Civil Engineering, Universidade da Coruña, 15071 A Coruña, \\ Spain
}

\begin{abstract}
Fuel efficiency and occupant safety are two of the most important concerns in the automotive industry nowadays. Encouraged by the importance of this field of study, this research attempts an improvement in the crashworthiness of a vehicle crash absorber. This component consists in a square hollow steel tube filled with a honeycomb structure made of glass-fiber reinforced polyamide. Surrogate-based optimization techniques are used. The three objective functions chosen — mass, absorbed energy and peak load —are approximated by two different models: multivariate adaptive regression splines and gaussian process (kriging). The thickness of both parts, the shape of the honeycomb and its height are selected as design variables. Two preliminary analyses of the specimen are performed: the computation of the interaction effect and a comparison of a hollow tube with the specimen. From the results of multi-objective crashworthiness optimization two Pareto frontiers are obtained, one for the absorbed energy and mass, and another one for the absorbed energy and peak load. The results achieved show great improvements on all objective functions compared to the original design. The peak load is reduced by $37 \%$ on a specimen with similar mass and absorbed energy, and the specific energy absorbed is increased by $39.5 \%$ for a specimen with a similar peak load to the one from the initial model.
\end{abstract}

Keywords: Crashworthiness, multi-objective optimization, surrogate methods, honeycomb structure, genetic algorithm

\footnotetext{
*Corresponding author. Tel: +34981 167 000; Fax: +34 981167170

Email addresses: javier.paz.mendez@udc.es (J. Paz), jdiaz@udc.es (J. Díaz), Iromera@udc.es (L. Romera), miguel . costas@udc.es (M. Costas)
} 


\section{Introduction}

Society's increasing demand for lower fuel consumption and higher safety for vehicle occupants has been recently conditioning the automotive industry. Car manufacturers are drawing their efforts into structural crashworthiness and vehicular lightweight, but these two opposite interests usually compete with each other. A trade-off between both criteria needs to be made in order to obtain vehicles with the best performance in both fields. As a result, $R \& D$ in crashworthiness has been gaining importance ever since, and managed to reduce fatalities considerably by $24.5 \%$ in the USA from 2006 to 2013 [1].

One procedure traditionally used for absorbing and dissipating the energy during an impact consists in bonding in-between the bumper and the car's chassis a hollow or ingeniously filled device, usually a hollow steel beam which collapses in axial crushing. This piece will absorb and dissipate through plastic deformation in the axial direction a large amount of the energy produced during the impact, thereby reducing the severity and consequences of the crash for the passengers. Nevertheless, not only the energy absorbed but also the mass and price of the element are factors taken into account during its design, seeing that weight-saving, fuel consumption and assembling price are other critical characteristics in car design.

J. M. Alexander [2] was the first to investigate in this topic, being able to obtain an approximate theoretical expression of the mean axial crushing force in thin-walled circular tubes back in the 1960s. Almost 20 years later, Wierzbicki and Abramowicz [3] developed the super folding element theory which investigated theoretical predictions for the axial mean crushing strength of rectangular tubes. In the experimental models from Abramowicz and Wierzbicki [4], Abramowicz and Jones [5], Pugsley [6], and Singace and Elsobky [7]; Alexander's tube was redesigned by shaping it with rectangular, circular and multi-corner sections; which was later axially crushed, loaded both statically and dynamically. They also carried out multiple studies dealing with the different collapse modes, obtaining an excellent resemblance between the theoretical predictions and the experimental results [8].

Although it had already become essential in aviation in the 1970s [9], it was not until the mid 1990s that crashworthiness extensively appeared in the car industry with Jones', White's and Abramowicz's experimental and theoretical analyses (of quasi-static axial crushing of top-hat and double-hat thin-walled sections) of car-usable steel sections [10, 11]. After having proved the outstanding strength and energy absorption of composite 
materials, car makers attempted to build cars using those exclusively [12]. This turned out to be extremely difficult due to the complexity of modeling these materials, especially by reason of delamination mechanisms [12]. As a result, research started focusing on the degradation processes of composite materials, like the work from Johnson and David [13], Israr et al. [14] or Jia et al. [15]. These reinforced materials have unstable and non-uniform collapsing modes that complement the ductile collapse mechanisms of the hollow metal structures studied in this article, which behave according to their geometry, thickness and inner ply configurations. As the research about using composite materials evolved [16, 17], so did the characteristics of the inner component. The filling of the metal tube is decisive in order to improve the crashworthiness behavior, with two variables to be chosen: the core material and its spatial configuration.

Regarding the material used for the filling part, many different options have been explored: natural and artificial cork [18, 19], polymer-based or aluminum-based artificial foams [20, 21] or metals like aluminum [22]. However, and since the beginning of the 2000s, carbon-fiber reinforced polymers (CFRP) and glass-fiber reinforced polymers (GFRP) are also used with exceptional results [23, 24]

As for the shape of the filling, it can be tight-compact (usually for cork and foam), a core of laminates, a honeycomb-like structure, or different optimized combinations. In the same way that the core bracing effect changes the buckling modes of a steel beam by reducing its buckling length, the collapse process of the steel tubes will be constrained by the composite material. Consequently, higher values of the total absorbed energy are reached, compared to the values obtained from crushing the separate parts [25]. As a consequence of this, the internal shape is as important as the material itself, with both thoroughly connected. There are different reasons that justify the use of a honeycomb as the filling shape. It has been deeply looked into, both theoretically and experimentally $[22,26-30]$, proving to be one of the best shapes. Most honeycomb structures are made of aluminum because of the lightweight and ductility of the material. Nevertheless, recent studies have started testing honeycombs made of GFRP and CFRP. Another tendency is filling the honeycomb with different materials, combining, for example, the progressive collapse of foams with the ductility of aluminum [31, 32].

The mean crushing strength of a square tube filled with a honeycomb structure is increased when compared to the empty one [33]. Therefore, complementing the steel tube with a core material translates into a better crashworthiness of the specimen, since the energy absorption values are higher and the overall weight of the car can be reduced. 
In order to find the best shape and sizing of the filling material, optimization has to be performed. The surrogate-based crashworthiness optimization began in the late 1990s, with Yamazaki and Han's work on square and circular tubes [34]. Other authors [35, 36] also began using this methods in vehicle parts or full car finite element models, obtaining significant improvements in the elements tested. As the computational power increased, so did the problem sizes and the accuracy of the results. In 2007, Hou et al. [26] optimized hexagonal metal tubes by maximizing the specific energy absorption (SEA), defined as the ratio between the absorbed energy and the specimen's mass, and minimizing the peak crushing load. The finite element model was run to obtain a full-factorial sampling, creating afterward the surrogate based model. Gu et al. [37] evaluated the performance of different surrogate methods considering the robustness of the method and its reliability. Most researchers consider the SEA as one of the objective functions for the optimization process. Nevertheless, the peak load is also monitored, since the usage of parts less stiff than the chassis protects the latter in low-energy impacts, remaining nearly undamaged.

This research attempts to take one step further the existing solutions for energy absorption and safety in the crashworthiness field. For this, a standard square steel tube is filled with a GFRP honeycomb structure, constituting a typical car crash absorber. It is afterward analyzed by using a nonlinear finite element code. The objective is finding a shape of this complete specimen which can greatly improve its crashworthiness capabilities. For the honeycomb, cell size, wall thickness, and total height are the three design variables; whereas only the wall thickness in the steel tube is variable. Different energy absorption parameters — conditioned by the variables of the specimen - were obtained from quasi-static and dynamic crushing analysis. This parameters are later used as objective functions and indicators to verify whether the designs comply with the initial expectations or not. Once the numerical results are obtained and processed, the optimized model is once again tested to corroborate the effectiveness in crashworthiness.

\section{Surrogate-based multi-objective optimization}

Crashworthiness optimization has to be approached by specifically considering the computational behavior of its functions. The first aspect to take into account is the problematic nature of the functions that have to be optimized. Since they originate from explicit computer simulations, they often are non-differentiable and intrinsically noisy functions; characteristics that would interfere with the proper functioning of gradient-based optimization methods. Another consideration refers to the computational cost. In models 
like the one in this research, equations involving plasticity, friction, failure and contact are evaluated constantly to provide solutions that resemble reality; but this comes with a drawback. Despite the innovations and high performance supercomputers available, evaluating one single design can take from hours to days, which suggests the need of an intelligent exploitation of the computer power.

As an answer to these problems, surrogate-based methods have been applied. The main idea behind these methods consists in replacing the expensive and complex physical model by another physical or mathematical model, the surrogate model, with the sole purpose of optimizing and modeling the first one. For this reason, the original objective functions $f_{i}$ are replaced by other functions easier to evaluate, $\hat{f}_{i}$. This surrogate model is faster when computed because it is usually constructed with more tractable functions. These functions, like n-th order polynomials or sets of basis functions such as splines or Gauss functions, avoid the singular, discontinuous, and non-differentiable points from the original model. The similarity of the approximation between the real and surrogate models will condition the results obtained in a later phase.

The first step for obtaining a surrogate model is defining a sampling plan. This sampling is crucial, since the adequacy of the points chosen will condition later on the suitability of the surrogate model. Full factorial sampling, which samples the model by creating a rectangular grid of points, is discarded in virtue of the high computational times required. Instead, the latin hypercube sampling (LHS) [38] was used. This stratified method, unlike the full factorial sampling, creates a set of data points that have homogeneous projections onto each variable axis, thus avoiding superimposed projections.

The second step consists in adjusting the proper functions for the data points obtained. Two strategies are considered:

Gaussian process (Kriging) It is one of the most common models in surrogate-based optimization. The approximation follows the structure

$$
\hat{f}_{G P}(\boldsymbol{x})=\boldsymbol{g}(\boldsymbol{x})^{T} \boldsymbol{\beta}+\varepsilon(\boldsymbol{x})
$$

where $\boldsymbol{x}$ is the current point, $\boldsymbol{g}(\boldsymbol{x})$ is the vector of basis functions at $\boldsymbol{x}$ and $\boldsymbol{\beta}$ is a vector containing the least squares estimates of the basis function coefficients. In this research, quadratic polynomials were used as the trend basis functions. The last term, $\varepsilon(\boldsymbol{x})$, is the stationary gaussian process error model, and it is used to correct the trend functions $\boldsymbol{g}(\boldsymbol{x})$. The stationary gaussian process error model, with a mean 
equal to 0 and a constant variance $\sigma^{2}$, contains a stationary autocorrelation function $r\left(\boldsymbol{x}, \boldsymbol{x}^{\prime}\right)$ so its autocovariance function is of the form (2). This error $\varepsilon(\boldsymbol{x})$ adjusts the trend function guaranteeing that at the given sampling points the overall error is zero.

$$
\sigma_{\hat{f}(x) \hat{f}\left(x^{\prime}\right)}=\sigma_{\hat{\varepsilon}(x) \hat{\varepsilon}\left(x^{\prime}\right)}=\sigma^{2} r\left(\boldsymbol{x}, \boldsymbol{x}^{\prime}\right)
$$

An anisotropic generalized exponential model (3) was used for the $r\left(\boldsymbol{x}, \boldsymbol{x}^{\prime}\right)$ function:

$$
r\left(\boldsymbol{x}, \boldsymbol{x}^{\prime}\right)=\exp \left(-\sum_{k=1}^{\Omega} \theta_{k}\left|x_{k}-x_{k}^{\prime}\right|^{\gamma}\right)
$$

where $\Omega$ is the number of input dimensions, $0<\gamma<2$ and $0<\theta_{k}$. For the gaussian correlation function $\gamma=2$, which is infinitely differentiable, the correlation parameters $\boldsymbol{\theta}$ are related to the correlation lengths $L_{k}$ by (4). The correlation lengths are analogous to standard deviation in the normal distribution.

$$
\theta_{k}=\frac{1}{2 L_{k}^{2}}
$$

Multivariate adaptive regression splines (MARS) This method uses multivariate adaptive regression splines [39] to fit the objective function, according to the next expression

$$
\hat{f}(\boldsymbol{x})=\sum_{m=1}^{M} a_{m} B_{m}(\boldsymbol{x}),
$$

where $B_{m}$ are the power basis functions (cubic splines were used in this research), $a_{m}$ the coefficients of the functions, and $M$ is the number of functions. The design space is partitioned into subregions, and for each subregion regression methods are applied to create a local surface fit in each one. The subregions are joined together to produce a $C^{2}$ continuous surface model. At first, the algorithm adjusts a single cubic spline to the design region. This design space is afterward divided into two subregions at a split point, called knot. The location of the knot is optimized so that the highest goodness of fit is obtained. With the design space divided in two subregions, a second spline is added to fit this new subregion. The process continues by adding knots and subsequently adjusting new splines until the maximum number of functions $M$ is reached. The model with $M$ splines enters a new phase in which each iteration removes a base function from the model. The function selected for removal needs to improve the most the fit of the model or, if that were not possible, 
harm the model the least. The only function that cannot be removed is the first spline, since its deletion could produce a hole in the model. At the end of this second phase, a new set of $M-1$ different models are obtained, and the algorithm now chooses the best one. This method is more thoroughly explained in [40].

The last step is the optimization itself. The method selected for this particular is the multi-objective genetic algorithm (MOGA) from the JEGA Library, developed by Eddy and Lewis [41]. This method performs Pareto optimization supporting general constraints as well as mixtures of real and discrete variables. The main working principle roots from the theory of the survival of the fittest. Unlike other multi-objective algorithms, MOGA has been specifically designed to avoid aggregating and scaling function values to transform the original problem into a single objective problem. The variables are encoded and referred to as chromosomes, and each digit of the variable is a gene. The first set of $N$ chromosomes is the initial population. The population members are ranked and then accepted or discarded depending on the number of other designs that dominate them. If the design is beyond the fitness frontier it is accepted and lives on to the next generation, where it will be evaluated again. Otherwise, it is discarded. Crossover and mutation are performed each generation to increase variability and dodge local minima. This way, even if a certain population tends to go to a certain local minimum, the member that suffers the mutation will move on to another point in the function and go on with the optimization approaching another minimum.

After the maximum number of generations or function evaluations is reached, the MOGA supplies the closest point to the utopia point (point that minimizes all objective functions) and generates the Pareto front. In the end, the set of points from the Pareto front constitutes the solution to the problem. This Pareto front points cannot improve the value of one objective function without worsening the value of at least another objective function.

\section{Model description}

\subsection{Specimen}

The specimen chosen for this research is a combination of two individual elements. The first one is a square hollow tube made from a standard S-275 J0H steel, with properties detailed in table 1 . The steel is modeled according to a Johnson-Cook strain-rate 
sensitive model, which yields to reliable solutions even if the material is subjected to various strain rates [42]. The model is a Mises plasticity model that also takes into account the strain-rate dependence, as well as temperature dependent effects. The constitutive equation of the steel model for yield stresses is:

$$
\sigma_{y}=\left[A+B\left(\varepsilon_{p}^{e f f}\right)^{P}\right](1+C \ln \dot{\varepsilon})\left[1-\left(T_{H}\right)^{Q}\right]
$$

where $A$ is the yielding stress, $B$ modifies the hardening law amplitude, $C$ affects the strain-rate dependency, $P$ changes the shape of the hardening law, and $Q$ adjusts the temperature dependency. Since the temperature dependency of the specimen is not taken into account, $\left(T_{H}\right)^{Q}=0$.

\begin{tabular}{ccccccccc}
\hline$E$ & $\sigma_{y}^{0}$ & $v$ & $\rho$ & $\mathrm{A}$ & $\mathrm{B}$ & $\mathrm{C}$ & $\mathrm{P}$ & $\dot{\varepsilon}_{0}$ \\
\hline $210 \mathrm{GPa}$ & $275 \mathrm{MPa}$ & 0.3 & $78.5 \mathrm{kN} / \mathrm{m}^{3}$ & $275 \mathrm{MPa}$ & $50 \mathrm{MPa}$ & 0.03 & 0.4 & 0.0001 \\
\hline
\end{tabular}

Table 1: S-275 J0H steel properties and Mises plasticity model values for equation (6). Taken from [43].

The second part is the inner reinforcement, which has a smaller section than the tube so it can be placed within the steel structure. It is made up of a honeycomb structure made of Ultramid A3WG10 BK00564 (BASF), a glass-fiber-reinforced-polyamide. Its mechanical properties have been extracted from the manufacturer's data sheets [44] and are shown in table 2 . These properties have also been implemented in the finite element model, as a linear, isotropic, elastic model with fragile failure. The short fibers of the composite are randomly chopped into a polyamide matrix, thus obtaining an isotropic behavior. For the failure criterion, it is considered that no further damage can occur to the material after the failure strain $\varepsilon_{u}$ is reached, so the elements are deleted after overpassing this value for the strain.

\begin{tabular}{ccccc}
\hline $\mathrm{E}$ & $v$ & $\rho$ & $\sigma_{u}$ & $\varepsilon_{u}$ \\
\hline $10.16 \mathrm{GPa}$ & 0.4 & $15.15 \mathrm{kN} / \mathrm{m}^{3}$ & $254 \mathrm{MPa}$ & $2.6 \%$ \\
\hline
\end{tabular}

Table 2: Material properties of Ultramid A3WG10 BK00564.

Since this investigation consists in a set of finite element analyses, the meshing of the model also needs to be detailed as it is an important factor for the results obtained. The mesh size varies according to each material and its attributes. In the steel tube, there 
has to be enough elements to allow the specimen to fold according to its natural crushing modes. This requires a moderate to high finite element density, but avoiding a very high density which would greatly increase computation times. The mesh size of the tube was set considering the previous work done by Costas et al. [19], where the mesh size was selected by taking into account the experimental folding process of a similar tube. In this research, a $4 \mathrm{~mm}$ mesh is implemented, trying to obtain an accurate steel folding process during the impact process. The honeycomb part has been meshed proportionally to the size of the cell, ranging from $1.6 \mathrm{~mm}$ to $3.5 \mathrm{~mm}$, so that every cell wall has at least two elements and no more than six. Figure 1 represents a model already meshed accordingly and with the actual thickness of the elements. Both parts consist of four-node shell elements with 3 integration points through their thickness and a reduced integration scheme.

\subsection{Design variables and objective functions}

The standard energy absorption device that will be used during the rest of the research is now defined. For the sizing of the specimen, there are two design parameters and four design variables (table 3). The two design parameters are the height of the tube, which takes a value of $250 \mathrm{~mm}$, and the edge of the square tube, equal to $100 \mathrm{~mm}$.

The design variables chosen for this research are:

- T1: Thickness of the steel sheets.

- T2: Thickness of the GFRP honeycomb reinforcement.

- D: Diagonal length of a single honeycomb cell.

- H1: Height of the GFRP honeycomb reinforcement.

Table 3 gives insight on the upper and lower bounds of each variable, as well as their initial values and to which specimen part they belong to. For a better understanding of the specimen, the measurements detailed in table 3 are depicted in figures 2 and 3 .

The objective of this research is to find a set of optimum designs of the specimen previously described according to three different objective functions. These functions are the absorbed energy $\left(E_{a}\right)$, the mass of the specimen $(m)$ and the peak load $\left(P_{\text {peak }}\right)$. The three metrics are obtained using a finite element simulation, where $E_{a}$ and $P_{\text {peak }}$ are obtained via the force-displacement curves. 


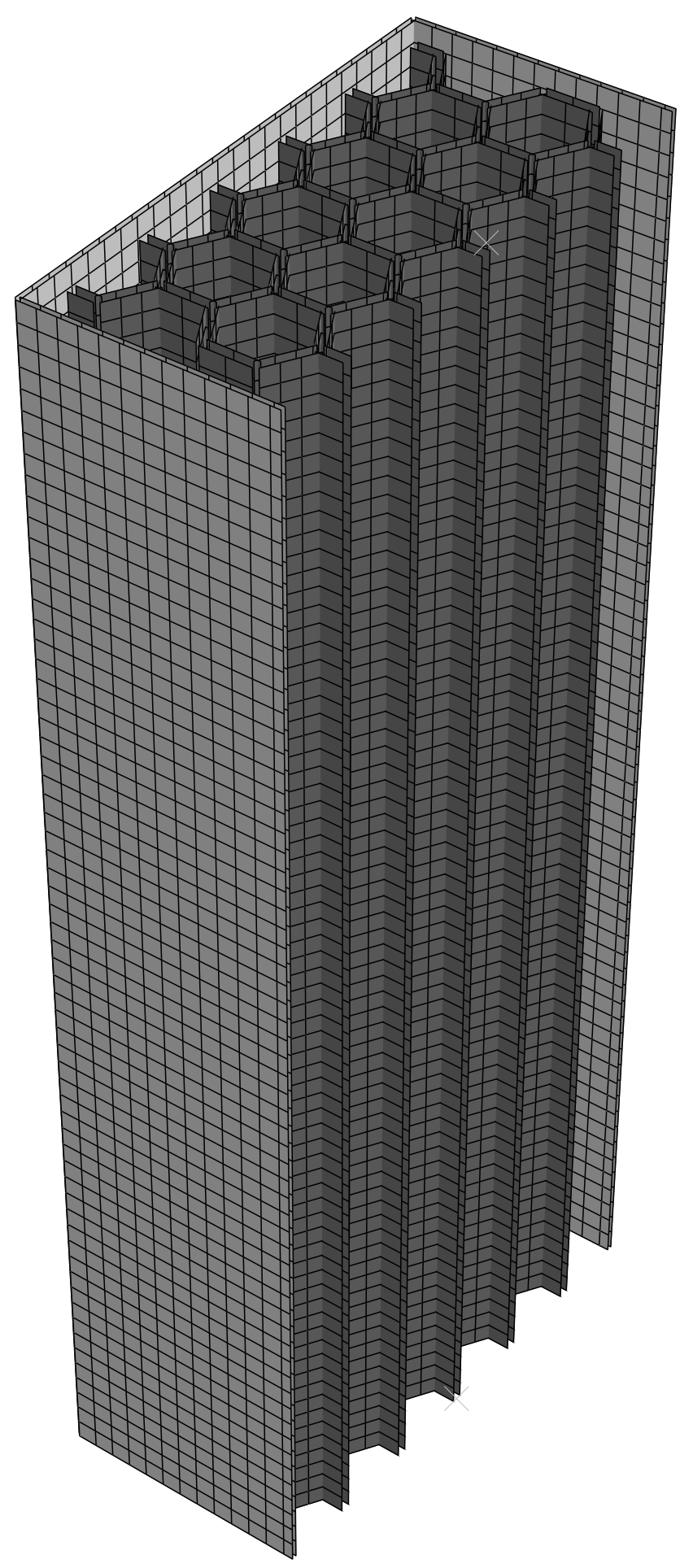

Figure 1: Three-dimensional cut of the finite element mesh of the specimen.

Before making any calculations with the force-displacement curve, and according to the specialized literature in crash analysis [45], a standard SAE 600 filter [46] is applied. This removes the high-frequency noise from the curve with a cutoff frequency of 1000 Hz. Once the filter is used, the direct integration of the resulting force-displacement curve yields to the $E_{a}$ :

$$
E_{a}=\int_{0}^{\delta} F(z) d z
$$




\begin{tabular}{lcrrr}
\hline & & \multicolumn{3}{c}{ Bounds } \\
\cline { 3 - 4 } Part & Design Variable & Lower & Upper & Initial Design \\
\hline Tube & T1 & 0.87 & 2.44 & 1.50 \\
\hline \multirow{3}{*}{ Honeycomb } & T2 & 1.00 & 3.00 & 1.50 \\
& $\mathrm{D}$ & 8.00 & 20.00 & 20.00 \\
& $\mathrm{H} 1$ & 220.00 & 250.00 & 250.00 \\
\hline
\end{tabular}

Table 3: Characteristics of design variables from figures 2 and 3. All dimensions in millimeters.

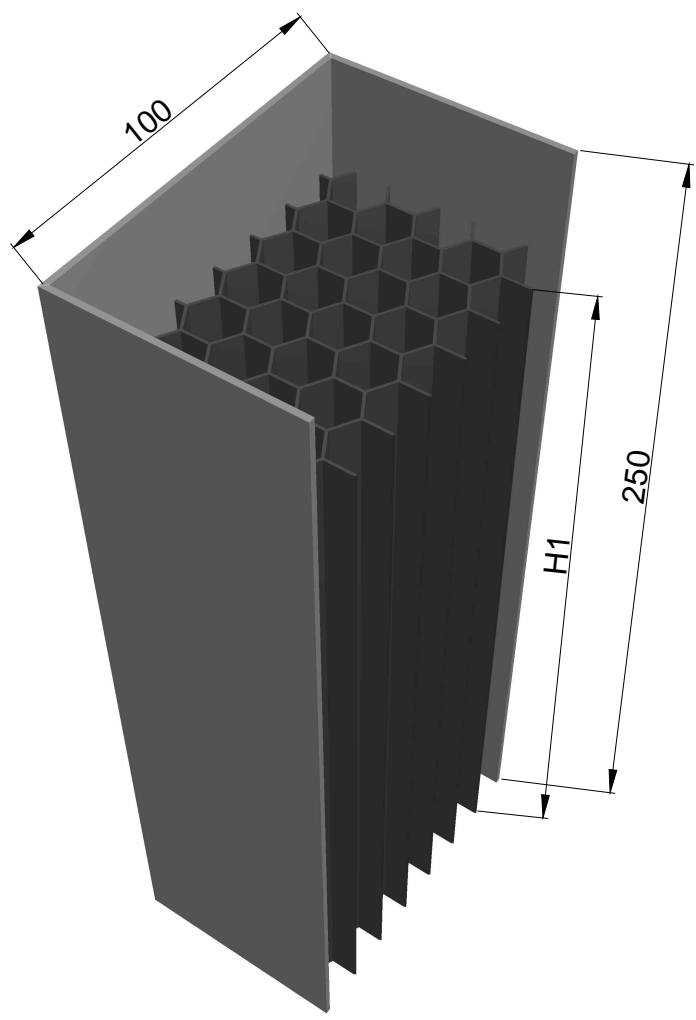

Figure 2: Three-dimensional cut of the specimen. All dimensions in millimeters.

with $\delta$ being the total axial crushing distance and $F(z)$ the value of the crushing force at the crushing length $z$.

The peak load $P_{\text {peak }}$ is defined as

$$
P_{\text {peak }}=\max \{F(z) \forall z \in[0, \delta]\}
$$

These three objective functions have not been randomly chosen. The first reason is the nature of the design and its aim to improve the crashworthiness of vehicles. The $E_{a}$ can be easily improved by increasing the thicknesses of the materials or making the honeycomb cells smaller, but these actions would harm the other two objective functions $m$ and $P_{\text {peak }}$, 


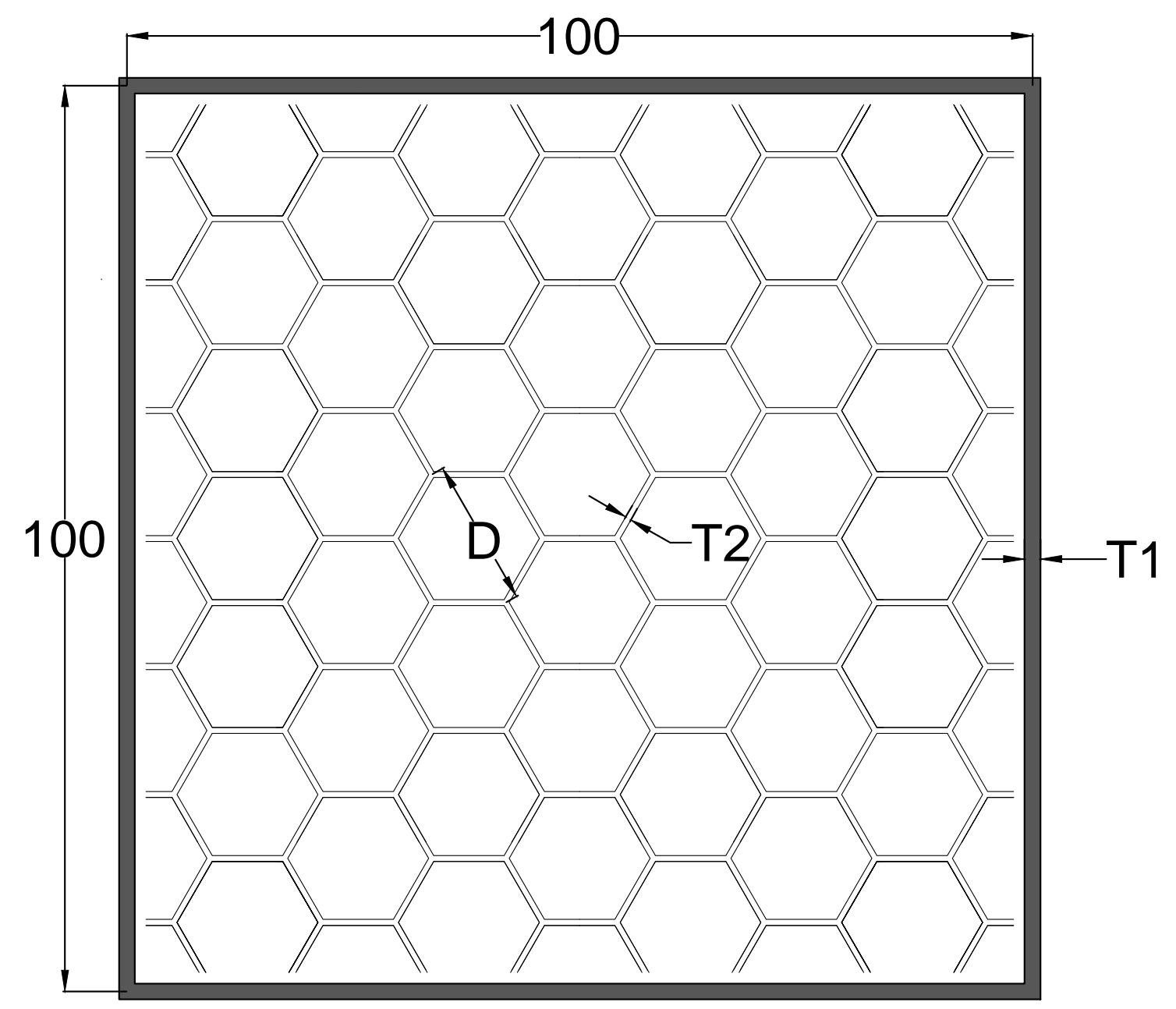

Figure 3: Top view of the specimen. All dimensions in millimeters.

increasing both of them. An increase in the mass of the specimen translates into a higher mass of the vehicle, increasing its fuel consumption and reducing its performance. On the other hand, an increase in the $P_{\text {peak }}$ means that the part is stiffer and needs more force to be crumpled, which translates into a dispersed damage throughout the whole chassis rather than local damage of the piece. Furthermore, the height of the honeycomb structure also has an effect on all three objective functions: besides augmenting the mass and the absorbed energy, since there is more material in the model, the initial peak force is increased as well. This happens because the initial peaks of the tube and the honeycomb occur at the same time, adding the forces of both peaks. When the height of the honeycomb is reduced, an offset between the peaks is observed, and the maximum total peak load is reduced.

The second reason for choosing these objective functions is purely computational. Traditionally, other researchers have swerved towards optimization of the specific energy absorption (SEA) and the load ratio (LR) [26]. The SEA function is defined as a ratio 
between $E_{a}$ and $m$ :

$$
S E A=\frac{E_{a}}{m}
$$

By contrast, the load ratio relates the $P_{\text {peak }}$ with the mean load $P_{m}$ as

$$
L R=\frac{P_{p e a k}}{P_{m}}
$$

The mean load is obtained as a ratio between the absorbed energy and the total crushing length $\delta$ :

$$
P_{m}=\frac{E_{a}}{\delta}
$$

The SEA and LR were also evaluated as objective functions, but it was found that during the optimization process these quantities have a more complicated comportment and a noisier nature for this model. Consequently, they were discarded from the optimization process and are only used as indicators to compare different specimens.

\subsection{Analysis settings}

The simulations have been run with the specimen being crushed between two rigid plates. One plate is fixed, and the other impacts the specimen at a constant speed of 64 $\mathrm{km} / \mathrm{h}(17.78 \mathrm{~m} / \mathrm{s})$. This maximum speed matches the Euro NCAP protocol for frontal impact testing [47]. When a maximum crushing length of $\delta=180 \mathrm{~mm}$ is reached the analysis stops. This limitation has been set in order to avoid the undesired bottom-out effect and contamination of the final energy absorption values.

The explicit module of the Abaqus 6.13 FEA package [48] was used for the evaluation of the samples. For this research, 600 data points have been used for the sampling, after considering the nature of the four design variables and the three objective functions. The complete crushing of each specimen was divided into 500 time steps, in which the FEA writes the variables' values. On the other hand, the surrogate based optimization procedure and the MOGA were implemented via DAKOTA framework in its version 5.4.1 [49]. The problems were first divided into 4 domains and each of them was computed in a separate processor with 3000 megabytes of RAM. All the complete analyses were run in a high performance computing (HPC) cluster with a theoretical peak performance of

\subsection{TFLOP's.}




\section{Results and discussion}

\subsection{Initial results}

The first calculations need to determine whether the objective functions selected comply with the initial expectations, since they will be used throughout the rest of the research. In order to judge the accuracy of the trend functions, the goodness of fit $R^{2}$, is looked into. This indicator is defined as

$$
R^{2}=\left(\frac{\sigma_{f \hat{f}}}{\sqrt{\sigma_{f}^{2} \sigma_{\hat{f}}^{2}}}\right)^{2}
$$

This has been done for all objective functions (figure 4), obtaining values equal or higher than 0.95 for the three selected objective functions. It can be seen that the $R^{2}$ is lower for SEA and LR despite increasing the number of maximum basis functions used. Consequently, the option with $E_{a}, m$ and $P_{\text {peak }}$ as objective functions was verified to be better than the one with SEA and LR.

This indicator is only useful for the MARS approximation, since kriging always has an error equal to zero in the sampling points and therefore the $R^{2}$ value is 1 .

In order to test the accuracy of the emulator we have evaluated for the three objective functions the root mean squared error (RMSE) metric, which is defined as

$$
R M S E=\sqrt{\frac{\sum_{i=0}^{M}\left(f_{i}-\hat{f}_{i}\right)^{2}}{M}} .
$$

This metric was evaluated together with a 10-fold cross-validation strategy in order to find an adequate number of bases for the MARS model. The cross-validation technique consist of splitting the sample of data into $k$ roughly equal subsets called folds. Later on, one of the subsets is removed and the model is fitted to the remaining $k-1$ subsets. The model is then compared to the data points in the fold that was discarded, allowing to test the model predictive capabilities.

If we call $\Upsilon$ to a mapping so that $\Upsilon:\{1, \ldots, n\} \rightarrow\{1, \ldots, k\}$, this mapping allocates the $n$ training points to one of the $k$ subsets. The value of the surrogate model obtained by removing the fold $\Upsilon_{i}$ is defined $\hat{f}^{-\Upsilon_{i}}(\boldsymbol{x})$. The cross-validation measure, used to estimate the error of the prediction, is 


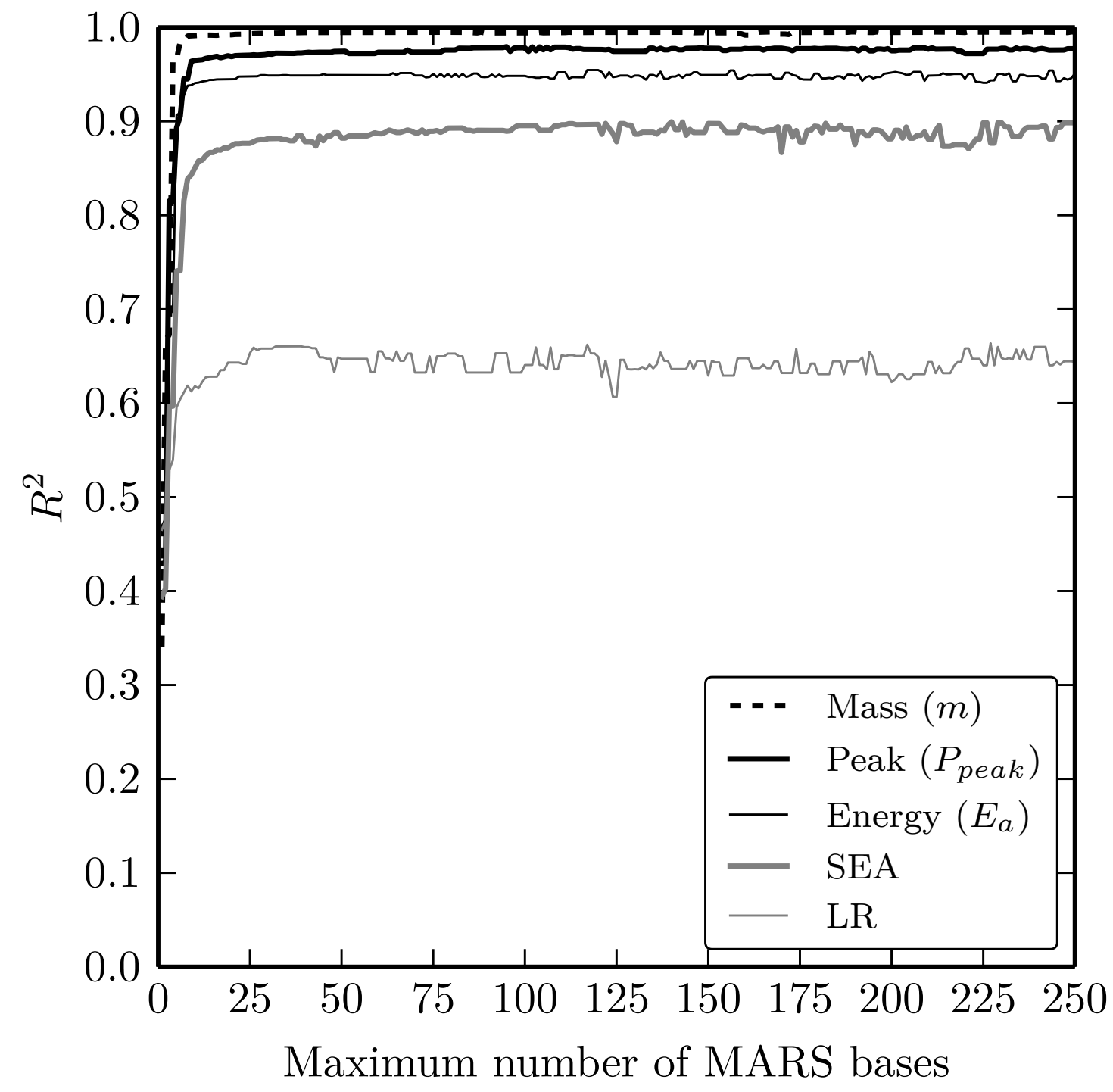

Figure 4: Variation of the $R^{2}$ indicator with the maximum number of MARS bases for different objective functions. 


$$
\varepsilon(c)=\frac{1}{2} \sum_{i=1}^{n}\left[f_{i}(\boldsymbol{x})-\hat{f}^{-\Upsilon_{i}}\left(\boldsymbol{x}^{i}, \boldsymbol{c}\right)\right]^{2} .
$$

Figure 5 shows the evolution of this indicator as the maximum number of MARS basis functions is increased. After an approximate maximum of 25 MARS basis functions, the mean absolute error stabilizes for all functions. This justifies using a maximum number of 48 MARS bases for the next optimization steps, since the error is not significantly reduced after this value.

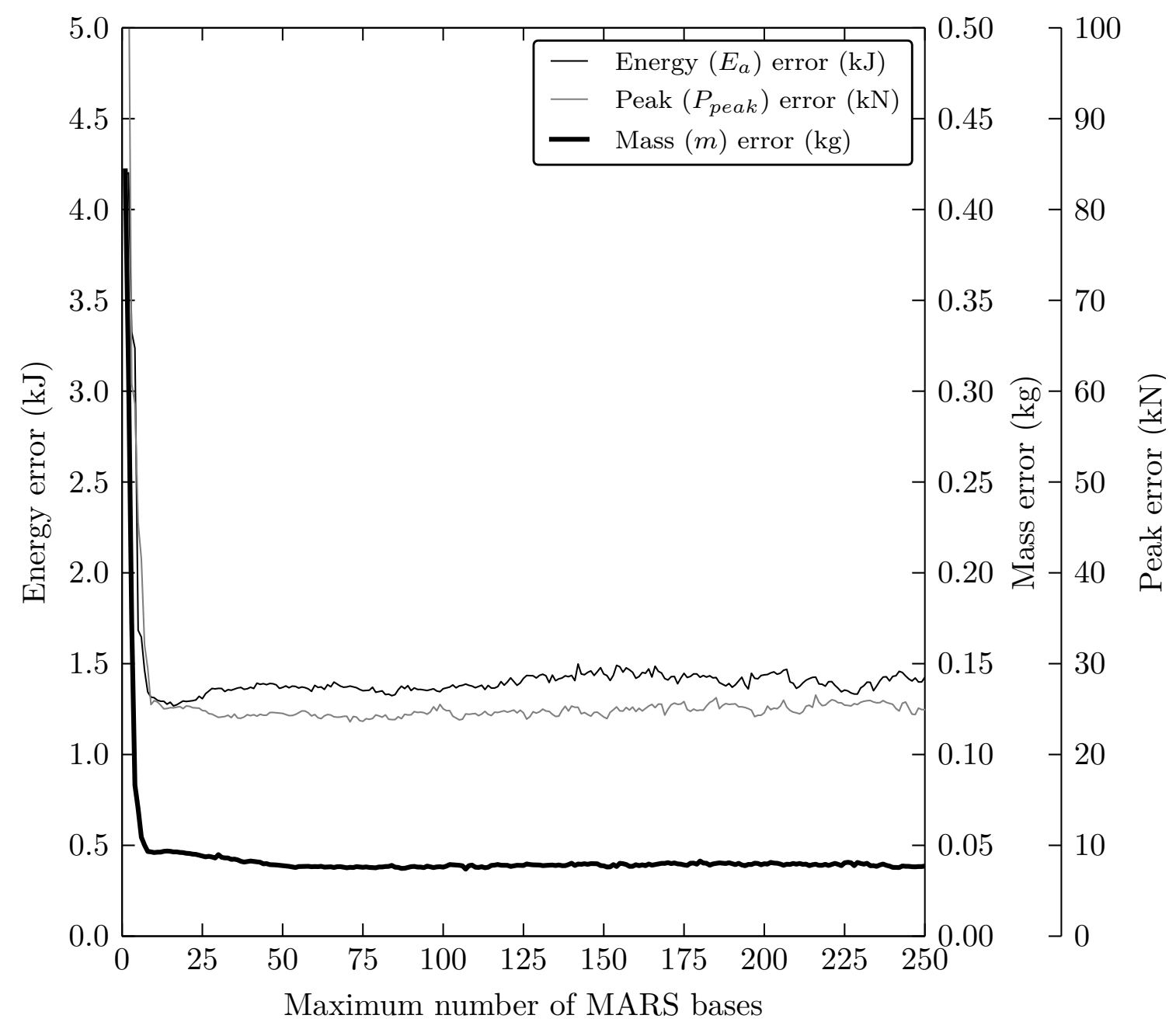

Figure 5: Influence of the maximum number of MARS maximum basis functions on the cross-validated mean absolute error for different objective functions.

Initial calculations with the initial design parameters shown in table 3 have been conducted to prove that the resulting capabilities of the combined specimen are better than those of both parts analyzed separately and summed afterward. For this, the initial model was analyzed as a whole, along with both of its separate components. The response of the detached components was then added and compared with the initial specimen, as it can be seen in figure 6 . The interaction effect accounts for an increase of $22 \%$ in the absorbed 


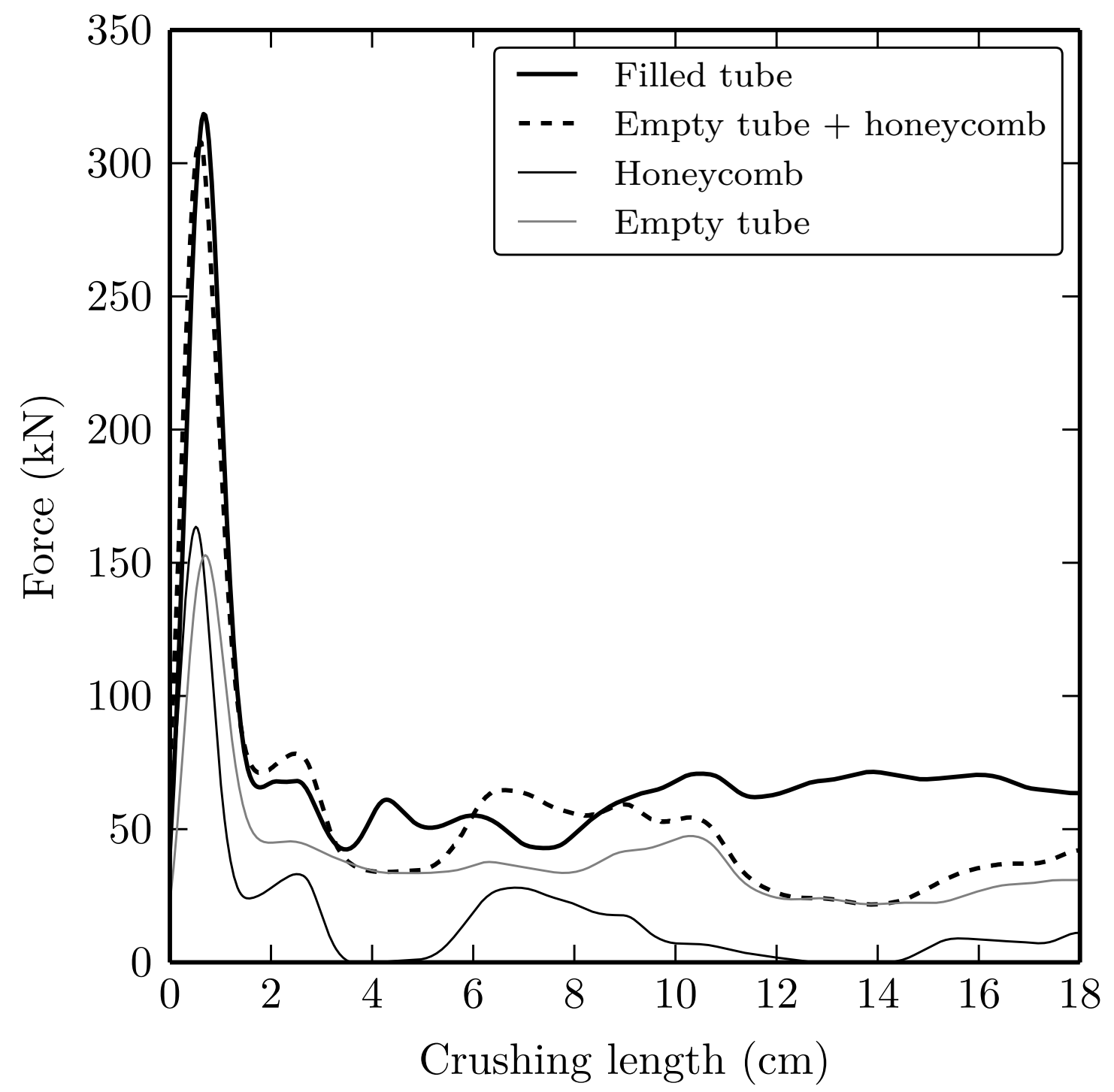

Figure 6: Force-displacement curves of the complete specimen, separate parts, and both parts added together.

energy in this example, with no significant variations in the peak load value. It is also noticeable that this effect is significant in the last part of the crushing of the specimen, after a crushing length of $9 \mathrm{~cm}$. Whereas the energy absorbed by both separate parts amounts to $3.19 \mathrm{~kJ}$ in the last $9 \mathrm{~cm}$ of the crushing process; the tube filled with the honeycomb absorbs $6.06 \mathrm{~kJ}$, nearly twice as much as the previous value. The more stable flattened response of the reinforced tube is produced by the higher energy dissipated compared to the empty tube, as more folds are developed during the crushing of the specimen (figure 7). Another important effect is the behavior of the honeycomb structure alone. During various crumpling steps the absorbed energy corresponding to this element is close to zero. This is caused by the absence of a casting, which would constrain the shattering of this piece and yield steadier results. 


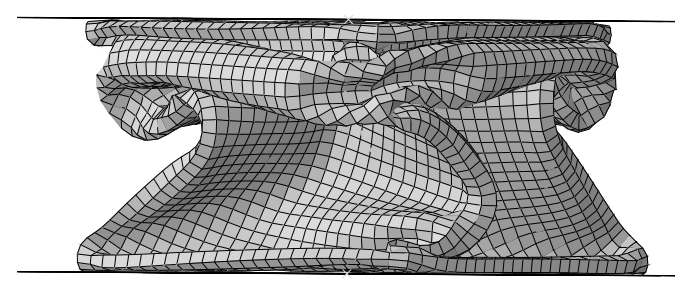

(a) Empty tube.

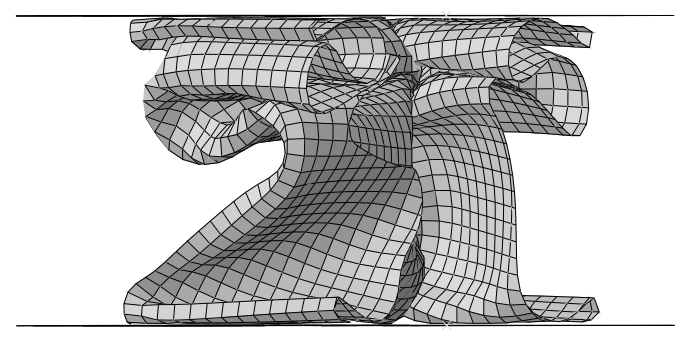

(c) Empty tube. Sectional cut

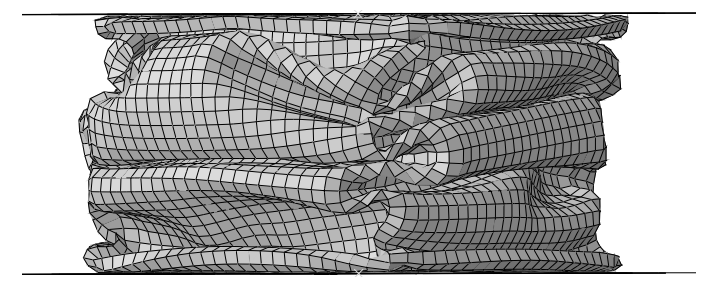

(b) Tube filled with GFRP-honeycomb structure.

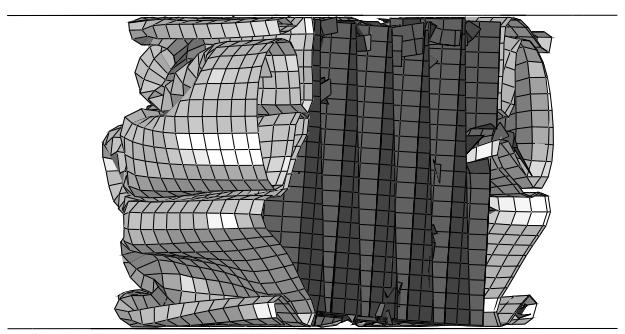

(d) Tube filled with GFRP-honeycomb structure. Sectional cut

Figure 7: Collapse modes of two square tubes.

Further tests have also been carried out with different specimens. With a defined value for the mass, two tubes were crushed. One of them was empty, and the other tube had thinner walls but had a honeycomb structure inside. The results show that for this certain design the energy absorption value is $29.1 \%$ higher and the peak load is only increased by $8.6 \%$ compared to the empty tube (figure 8 ). This proves that filling the tube with a honeycomb structure can greatly improve the crashworthiness of the specimen without increasing the mass and, therefore, harming fuel consumption.

With all preliminary considerations taken into account, and seeing the improvement of both objective functions, the specimen is declared fit for analysis and undergoes the optimization process.

\subsection{Optimization results}

After running the optimization algorithm, the MOGA outputs a set of points that conform the Pareto front. There are two sets Pareto fronts, one obtained with MARS (figures $9 \mathrm{a}$ and 9b), and another one obtained using a Gaussian process (figures 9c and 9d). As it can be seen in the figures, the Pareto fronts obtained with the Gaussian process have a less defined outline and the frontier is less clear. It is also noticeable that the Pareto sets from figures $9 \mathrm{~b}$ and $9 \mathrm{~d}$ have a noisier nature, caused by the strong slope discontinuities of the peak objective function and, in the case of the MARS model, because of the lower $R^{2}$ value in the approximation. Despite this, both Pareto fronts obtained with MARS have a clearly defined boundary, establishing a set of optimum designs. Therefore, this Pareto 


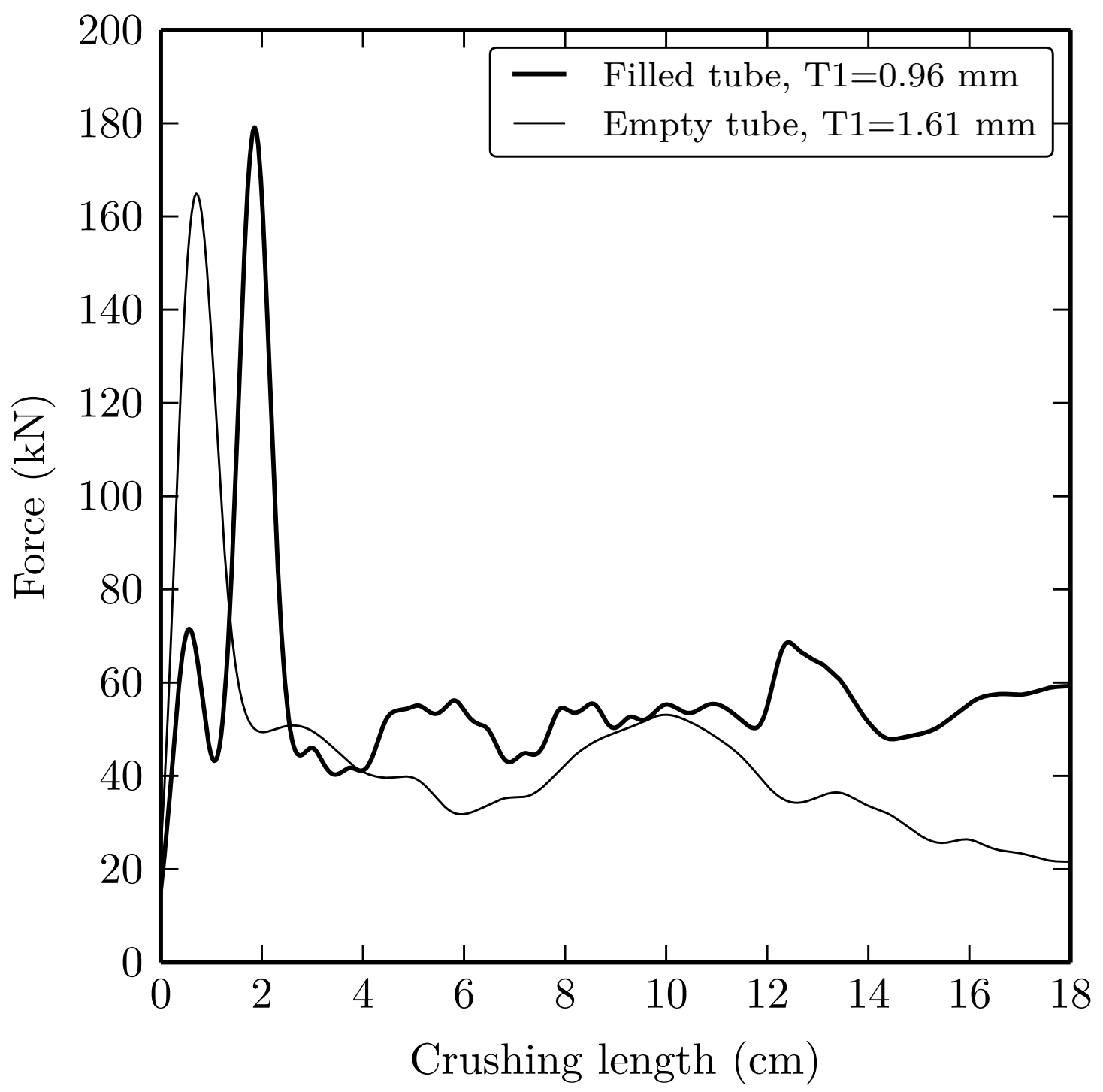

Figure 8: Force-displacement curves of the complete specimen and a hollow tube with equal mass.

fronts are used during the rest of the research.

The two following tables show the values for the design variables and objective functions of different points from the Pareto front. Table 4 corresponds to points from the energy-mass Pareto front, and table 5 to points from the energy-peak load Pareto front.

The energy-mass Pareto front has a quasi-linear shape throughout the whole design space. Also, it is noted that the SEA indicator varies throughout the frontier, ranging from $6.4 \mathrm{~kJ} / \mathrm{kg}$ for low energy values, to approximately $11 \mathrm{~kJ} / \mathrm{kg}$ at the other end of the front. Compared to the initial specimen, which had a SEA of $7.6 \mathrm{~kJ} / \mathrm{kg}$, all models in the Pareto front that absorb more than $11 \mathrm{~kJ}$ have a higher SEA value. The small portion of designs that have a lower SEA confirm that most specimens improve the capabilities of the initial design. As seen in table 4, in order to increase the energy absorbed by the 


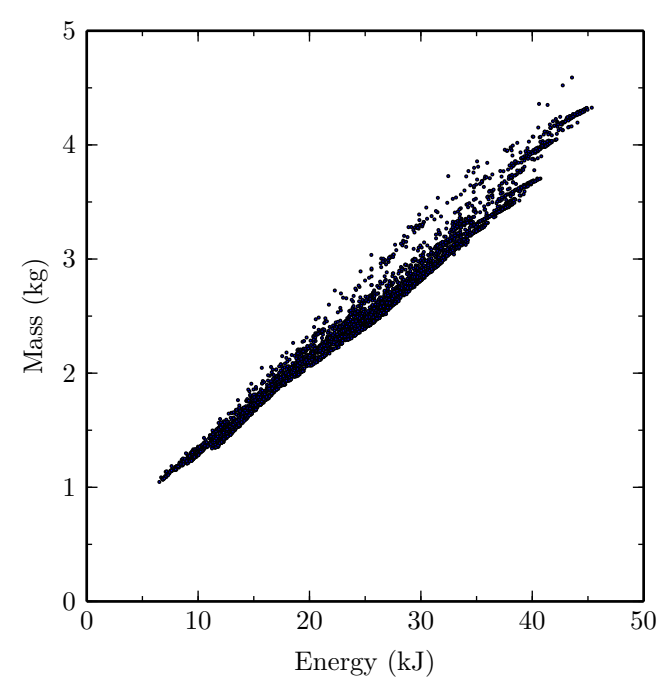

(a) Pareto front for the absorbed energy and the mass obtained from the MOGA on 48bases MARS model.

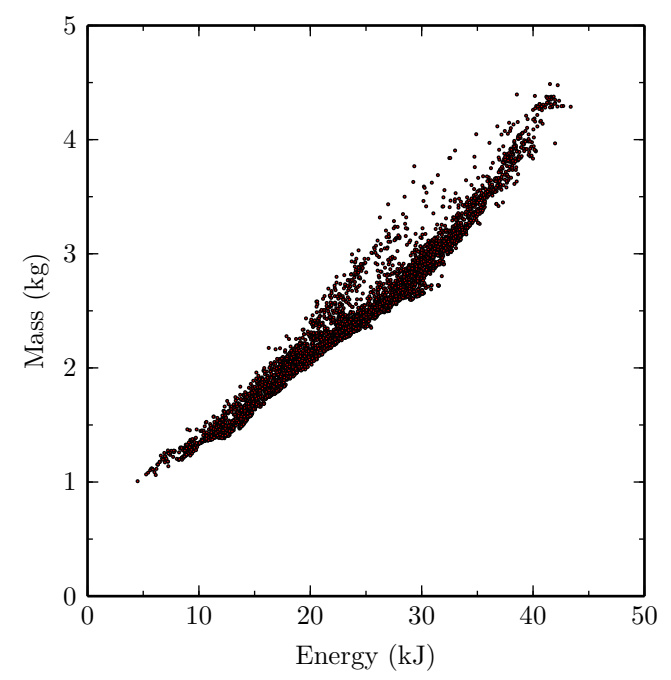

(c) Pareto front for the absorbed energy and the mass obtained from the MOGA with a Gaussian process.

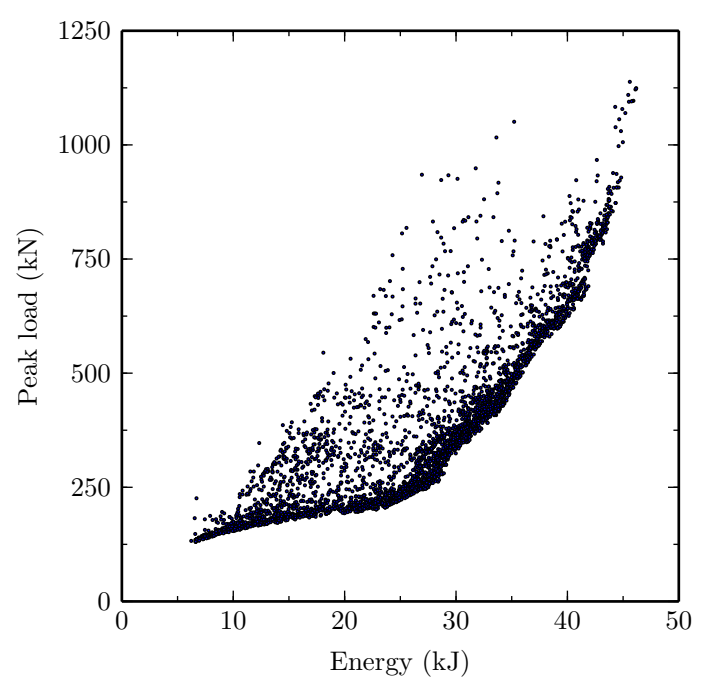

(b) Pareto front for absorbed energy and the peak load obtained from the MOGA on 48bases MARS model.

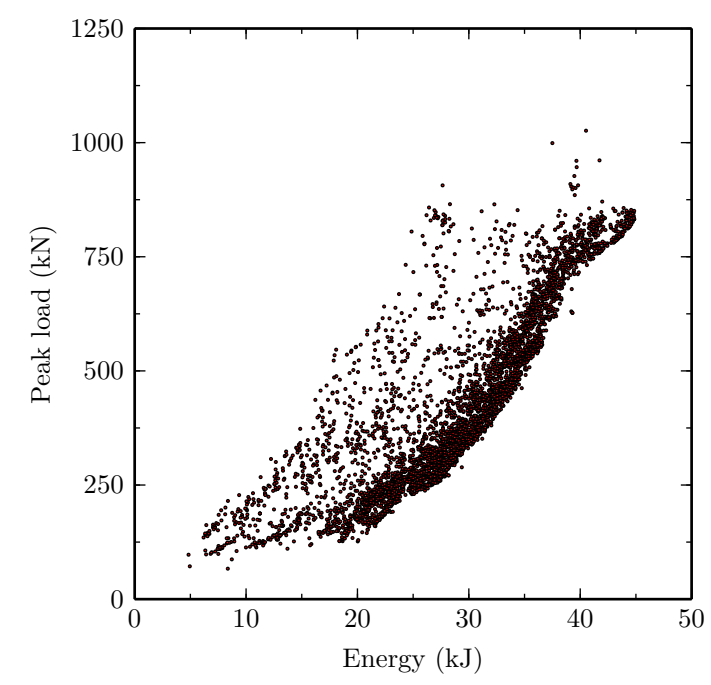

(d) Pareto front for absorbed energy and the peak load obtained from the MOGA with a Gaussian process.

Figure 9: Pareto fronts obtained from the MOGA. 


\begin{tabular}{|c|c|c|c|c|c|c|}
\hline \multicolumn{4}{|c|}{ Design variables (mm) } & \multicolumn{2}{|c|}{ Objective functions } & \multirow{2}{*}{$\frac{\text { Indicator }}{\text { SEA }(\mathrm{kJ} / \mathrm{kg})}$} \\
\hline $\mathrm{D}$ & $\mathrm{T} 1$ & $\mathrm{~T} 2$ & $\mathrm{H}$ & $E_{a}(\mathrm{~kJ})$ & $\mathrm{m}(\mathrm{kg})$ & \\
\hline 18.55 & 0.92 & 1.03 & 247.78 & 6.86 & 1.07 & 6.41 \\
\hline 11.50 & 0.92 & 1.43 & 247.78 & 14.83 & 1.64 & 9.04 \\
\hline 11.48 & 2.13 & 1.03 & 247.67 & 23.48 & 2.29 & 10.25 \\
\hline 11.50 & 2.38 & 1.56 & 245.73 & 30.34 & 2.84 & 10.68 \\
\hline 11.50 & 2.33 & 2.43 & 247.67 & 36.70 & 3.37 & 10.89 \\
\hline 11.47 & 2.36 & 2.90 & 247.78 & 40.73 & 3.71 & 10.99 \\
\hline
\end{tabular}

Table 4: Points from the energy-mass Pareto front shown in figure 9a.

\begin{tabular}{ccccccc}
\hline \multicolumn{3}{c}{ Design variables $(\mathrm{mm})$} & & \multicolumn{2}{c}{ Objective functions } \\
\cline { 1 - 2 } \cline { 7 - 7 } $\mathrm{D}$ & $\mathrm{T} 1$ & $\mathrm{~T} 2$ & $\mathrm{H}$ & & $E_{a}(\mathrm{~kJ})$ & $P_{\text {peak }}(\mathrm{kN})$ \\
\hline 19.89 & 0.96 & 1.10 & 233.84 & & 6.62 & 130.44 \\
14.80 & 1.80 & 1.01 & 235.18 & & 15.55 & 179.55 \\
14.48 & 2.44 & 1.02 & 235.08 & & 23.66 & 205.74 \\
14.29 & 2.44 & 1.63 & 235.58 & & 28.91 & 280.62 \\
14.21 & 2.44 & 2.72 & 234.27 & & 34.53 & 440.08 \\
10.73 & 2.44 & 2.94 & 235.92 & & 41.88 & 690.49 \\
8.21 & 2.44 & 2.97 & 238.46 & & 44.83 & 928.50 \\
\hline
\end{tabular}

Table 5: Points from the energy-peak load Pareto front shown in figure 9b.

specimen the cell size is reduced, both thicknesses are increased, and the honeycomb height tends to match that of the steel tube. All of this also leads to a higher mass of the object, but increasing the SEA indicator.

The energy-peak load Pareto front starts with a linear tendency, and between the energy values of $19 \mathrm{~kJ}$ and $23 \mathrm{~kJ}$ the frontier becomes nearly horizontal. This means that in this small range the peak load is nearly stagnant even when the energy increases, an effect that was pursued in this research. After that, the frontier becomes steeper, so that a small increase of the absorbed energy demands a high peak load value increase, an undesirable effect for the model.

\subsection{Optimum points}

The initial design proposed in table 3 was analyzed and its results will be used as a reference for the optimum designs.

The algorithm used also provides the closest points to the utopia point, which constitute the optimum designs. Two optimum designs, 1 and 2, were run again to obtain the force-displacement curves and they were later on compared to the initial design, as shown in table 6. 


\begin{tabular}{ccrrr}
\hline & & & \multicolumn{2}{c}{ Optimum design (Improvement) } \\
\cline { 3 - 5 } Parameter type & Parameter & Initial design & 1 & 2 \\
\hline \multirow{3}{*}{ Design variable } & $\mathrm{D}(\mathrm{mm})$ & 20.00 & 13.13 & 13.31 \\
& $\mathrm{~T} 1(\mathrm{~mm})$ & 1.50 & 1.44 & 2.44 \\
& $\mathrm{~T} 2(\mathrm{~mm})$ & 1.50 & 1.03 & 1.53 \\
& $\mathrm{H}(\mathrm{mm})$ & 250.00 & 222.53 & 235.63 \\
\hline \multirow{3}{*}{ Objective function } & $E_{a}(\mathrm{~kJ})$ & 13.02 & $13.27(1.9 \%)$ & $29.50(126.6 \%)$ \\
& $m(\mathrm{~kg})$ & 1.72 & $1.68(2.3 \%)$ & $2.79(-62.2 \%)$ \\
& $P_{\text {peak }}(\mathrm{kN})$ & 318.47 & $200.77(37.0 \%)$ & $298.67(6.3 \%)$ \\
\hline Indicator & $\mathrm{SEA}(\mathrm{kJ} / \mathrm{kg})$ & 7.57 & $7.90(4.4 \%)$ & $10.56(39.5 \%)$ \\
\hline
\end{tabular}

Table 6: Comparison between initial design and optimum design.

The initial design has been optimized obtaining a peak load $37 \%$ smaller than the original one in the optimum design 1, with similar mass and energy absorption. It can be seen that the honeycomb height approaches the minimum value allowed, $220 \mathrm{~mm}$, in order to reduce the maximum peak load. The thickness of the honeycomb also tends to the lower boundary value, with the other variables in the middle part of the design spectrum.

The optimum design 2 has a maximum peak load nearly as high as that of the initial design, but with a higher specific energy absorption. Even though the mass is increased by $62.2 \%$, the improvement of $126.6 \%$ in the energy absorption yields a SEA $39.5 \%$ higher than the one obtained for the initial design. In this model the steel thickness tends towards the maximum value, showing that an increase in the mass of the steel provides high specific energy absorption values. The other variables are in the middle of the design range, slightly higher than the values for the optimum design 1.

Figure 10 shows how the peak load from the initial design —which is the sum of the peak load from the tube and the honeycomb - splits into two peaks in the optimum designs, yielding to similar energy absorption values with smaller peak loads. The offset in the peaks is caused by the different height of both parts. After the peak load produced by the tube, the force decreases. When the peak load from the honeycomb occurs, the total force is not the sum of both peaks but the force from the honeycomb peak load and a lower value from the crushing of the tube. This second peak load is higher that the first one, which justifies the low honeycomb thicknesses of both optimum designs. If the value of the variable T2 were higher, the second peak load would be increased, and therefore so would the overall peak load. A utopian design would have both peaks equal, maximizing the area under the force-displacement curve and minimizing the maximum peak load. 


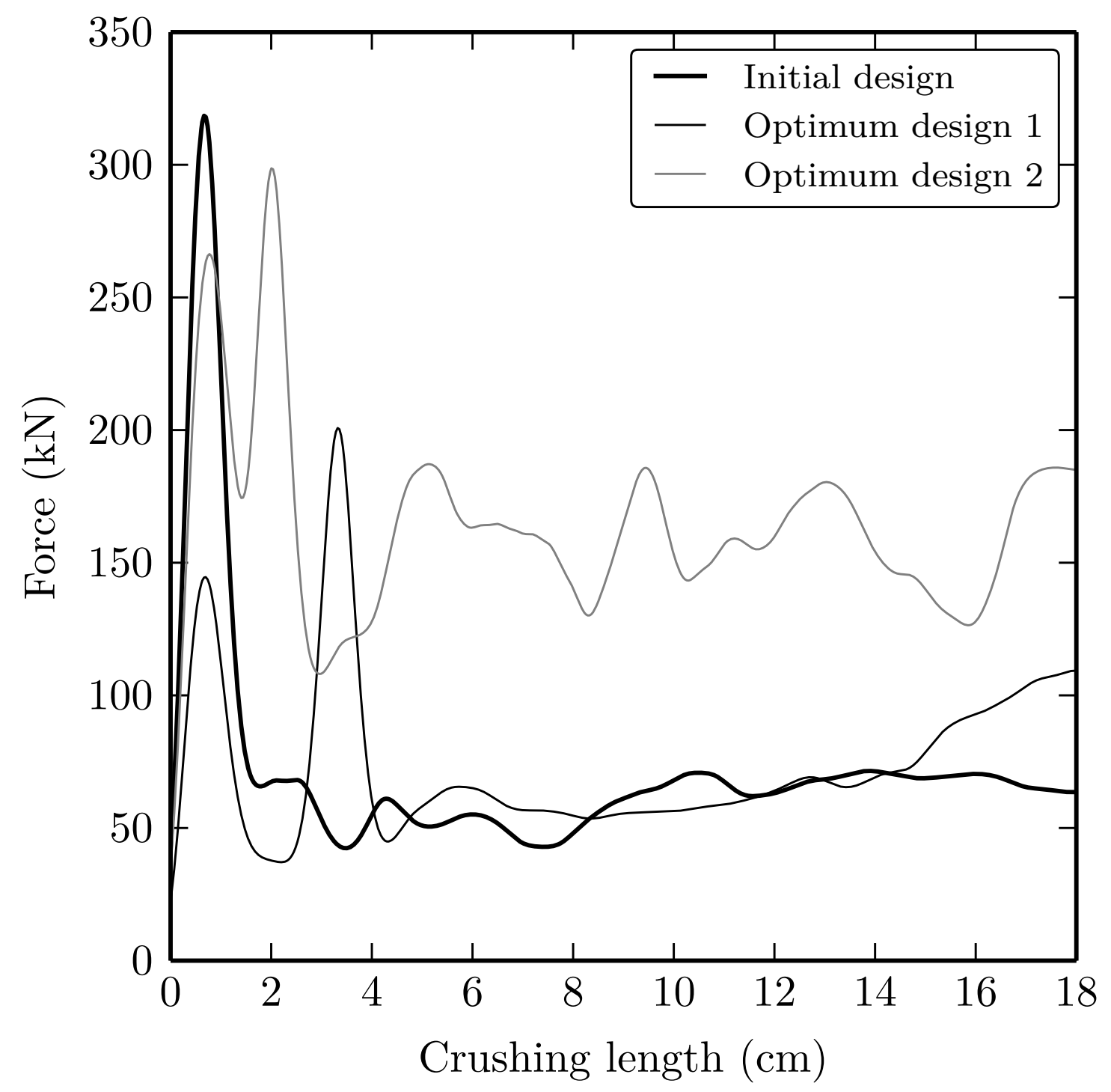

Figure 10: Force-displacement curves of the initial and optimum specimens.

\section{Conclusions}

This paper attempts an improvement in the crashworthiness of a traditional energy absorption device, in order to increase fuel efficiency and occupant safety. For this, multiobjective structural optimization of a honeycomb-filled square tube are performed. Three objective functions - mass, absorbed energy and peak load — have been selected because of their contrasting nature and the high goodness of fit during the approximation. An initial study was carried out to determine the approximate number of multi-adaptive regression splines basis functions needed to resemble accurately the objective functions that had to be optimized. Pareto optimization was performed with a multi-objective genetic algorithm, obtaining two Pareto fronts. Results show that the initial design has been optimized obtaining a peak load $37 \%$ smaller than the peak load from the initial design, with similar mass and energy absorption. The initial design was also improved with a model 
that has a specific energy absorption 39.5\% higher and a slightly smaller peak.

An initial model, the initial specimen, was selected as a reference to compare with the optimum designs which would be obtained. Also, some preliminary tests were carried out with this specimen. We proved that the interaction effect for the initial specimen accounts for $22 \%$ of the energy absorption, with no significant effects on the initial peak load. Another preliminary experiment compared the performance between an empty hollow tube and a specimen with equal mass and a glass-fiber reinforced polymer honeycomb structure inside. The latter option had an energy absorption $29.1 \%$ higher with a peak load increased by only $8.6 \%$ compared to the empty hollow tube.

The Pareto frontiers were obtained with MARS and a Gaussian process. The MARS model proved to be more adequate in this research, since it yields clearer and more stable Pareto fronts.

The energy-peak load Pareto front has a noisier nature than the energy-mass one since the peak load function has strong slope discontinuities and is approximated with a lower $R^{2}$ value than the mass function. The design variables from this model with the most influence in the peak load value are the honeycomb height and the honeycomb thickness, which greatly reduce the peak load as their value is also lowered.

The energy-mass Pareto front is clearer than the other Pareto front, being almost linear. The specific energy absorption increases as the absorbed energy and mass increase, obtaining values of up to $11 \mathrm{~kJ} / \mathrm{kg}$; when the initial model had a specific energy absorption value of $7.6 \mathrm{~kJ} / \mathrm{kg}$. The points from the energy-mass Pareto front tend to high values of the honeycomb part, therefore producing high values for the peak. Nevertheless, a reduction of this design variable would generate models with high energy absorption and lower peak load than the original model.

\section{Acknowledgments}

The research leading to these results has received funding from the Galician Regional Goverment (Xunta de Galicia) under the plan INCITE09, project reference 09DPI01118PR and GRC2013-056. The authors fully acknowledge the support received.

[1] National Highway Traffic Safety Administration - NHTSA, Early Estimate of Motor Vehicle Traffic Fatalities for the First Half (Jan-Jun) of 2013, Tech. Rep., U.S. Department of Transportation, 2013. 
[2] J. M. Alexander, An approximate analysis of the collapse of thin cylindrical shells under axial loading, Quarterly Journal of Mechanics and Applied Mathematics 13 (1) (1960) 10-15.

[3] T. Wierzbicki, W. Abramowicz, On the crushing mechanics of thin-walled structures, Journal of Applied Mechanics, Transactions ASME 50 (4 a) (1983) 727-734.

[4] W. Abramowicz, T. Wierzbicki, Axial crushing of multicorner sheet metal columns, Journal of Applied Mechanics, Transactions ASME 56 (1) (1989) 113-120.

[5] W. Abramowicz, N. Jones, Dynamic progressive buckling of circular and square tubes, International Journal of Impact Engineering 4 (4) (1986) 243-270.

[6] A. Pugsley, On the crumpling of thin tubular struts, Quarterly Journal of Mechanics and Applied Mathematics 32 (1) (1979) 1-7.

[7] A. Singace, H. Elsobky, Further experimental investigation on the eccentricity factor in the progressive crushing of tubes, International Journal of Solids and Structures 33 (24) (1996) 3517-3538.

[8] W. Abramowicz, N. Jones, Transition from initial global bending to progressive buckling of tubes loaded statically and dynamically, International Journal of Impact Engineering 19 (5-6) (1997) 415-437.

[9] A. Madayag, FAA general aviation crashworthiness program, SAE Technical Papers

[10] M. White, N. Jones, Experimental quasi-static axial crushing of top-hat and doublehat thin-walled sections, International Journal of Mechanical Sciences 41 (2) (1999) 179-208.

[11] M. White, N. Jones, W. Abramowicz, A theoretical analysis for the quasi-static axial crushing of top-hat and double-hat thin-walled sections, International Journal of Mechanical Sciences 41 (2) (1999) 209-233.

[12] A. Mamalis, D. Manolakos, G. Demosthenous, M. Ioannidis, The static and dynamic axial collapse of fibreglass composite automotive frame rails, Composite Structures 34 (1) (1996) 77-90. 
[13] A. Johnson, M. David, Failure mechanisms and energy absorption in composite elements under axial crush, Key Engineering Materials 488-489 (2012) 638-641.

[14] H. Israr, S. Rivallant, J. Barrau, Experimental investigation on mean crushing stress characterization of carbon-epoxy plies under compressive crushing mode, Composite Structures 96 (2013) 357-364.

[15] X. Jia, G. Chen, Y. Yu, G. Li, J. Zhu, X. Luo, C. Duan, X. Yang, D. Hui, Effect of geometric factor, winding angle and pre-crack angle on quasi-static crushing behavior of filament wound CFRP cylinder, Composites Part B: Engineering 45 (1) (2013) $1336-1343$.

[16] G. Jacob, J. Fellers, J. Starbuck, S. Simunovic, Crashworthiness of automotive composite material systems, Journal of Applied Polymer Science 92 (5) (2004) 32183225 .

[17] J. Huang, X. Wang, Numerical and experimental investigations on the axial crushing response of composite tubes, Composite Structures 91 (2) (2009) 222-228.

[18] M. Paulino, F. Teixeira-Dias, C. Gameiro, J. Cirne, Hyperelastic and dynamical behaviour of cork and its performance in energy absorption devices and crashworthiness applications, International Journal of Materials Engineering Innovation 1 (2) (2009) 197-234.

[19] M. Costas, J. Díaz, L. Romera, S. Hernández, A. Tielas, Static and dynamic axial crushing analysis of car frontal impact hybrid absorbers, International Journal of Impact Engineering 62 (2013) 166-181.

[20] M. Langseth, O. Hopperstad, A. Hanssen, Crash behaviour of thin-walled aluminium members, Thin-Walled Structures 32 (1-3) (1998) 127-150.

[21] J. Bi, H. Fang, Q. Wang, X. Ren, Modeling and optimization of foam-filled thinwalled columns for crashworthiness designs, Finite Elements in Analysis and Design 46 (9) (2010) 698-709.

[22] G. Sun, G. Li, M. Stone, Q. Li, A two-stage multi-fidelity optimization procedure for honeycomb-type cellular materials, Computational Materials Science 49 (3) (2010) 500-511. 
[23] H. Saito, I. Kimpara, Evaluation of impact damage mechanism of multi-axial stitched CFRP laminate, Composites Part A: Applied Science and Manufacturing 37 (12) (2006) 2226-2235.

[24] H. Hamada, S. Ramakrishna, A FEM method for prediction of energy absorption capability of crashworthy polymer composite materials, Journal of Reinforced Plastics and Composites 16 (3) (1997) 226-242.

[25] H.-W. Song, Z.-M. Wan, Z.-M. Xie, X.-W. Du, Axial impact behavior and energy absorption efficiency of composite wrapped metal tubes, International Journal of Impact Engineering 24 (4) (2000) 385-401.

[26] S. Hou, Q. Li, S. Long, X. Yang, W. Li, Design optimization of regular hexagonal thin-walled columns with crashworthiness criteria, Finite Elements in Analysis and Design 43 (6-7) (2007) 555-565.

[27] L. Aktay, A. Johnson, B.-H. Kröplin, Numerical modelling of honeycomb core crush behaviour, Engineering Fracture Mechanics 75 (9) (2008) 2616-2630.

[28] H. Yin, G. Wen, N. Gan, Crashworthiness design for honeycomb structures under axial dynamic loading, International Journal of Computational Methods 8 (4) (2011) $863-877$.

[29] H. Yin, G. Wen, S. Hou, K. Chen, Crushing analysis and multiobjective crashworthiness optimization of honeycomb-filled single and bitubular polygonal tubes, Materials and Design 32 (8-9) (2011) 4449-4460.

[30] J. Schultz, D. Griese, J. Ju, P. Shankar, J. Summers, L. Thompson, Design of honeycomb mesostructures for crushing energy absorption, Journal of Mechanical Design, Transactions of the ASME 134 (7).

[31] A. Alavi Nia, M. Sadeghi, The effects of foam filling on compressive response of hexagonal cell aluminum honeycombs under axial loading-experimental study, Materials and Design 31 (3) (2010) 1216-1230.

[32] S. Santosa, T. Wierzbicki, Crash behavior of box columns filled with aluminum honeycomb or foam, Computers and Structures 68 (4) (1998) 343-367.

[33] H. Zarei, M. Kröger, Crashworthiness optimization of empty and filled aluminum crash boxes, International Journal of Crashworthiness 12 (3) (2007) 255-264. 
[34] K. Yamazaki, J. Han, Maximization of the crushing energy absorption of tubes, Structural Optimization 16 (1) (1998) 37-46.

[35] P. Marklund, L. Nilsson, Optimization of a car body component subjected to side impact, Structural and Multidisciplinary Optimization 21 (5) (2001) 383-392.

[36] J. Sobieszczanski-Sobieski, S. Kodiyalam, R. Yang, Optimization of car body under constraints of noise, vibration, and harshness (NVH), and crash, Structural and Multidisciplinary Optimization 22 (4) (2001) 295-306.

[37] X. Gu, G. Sun, G. Li, L. Mao, Q. Li, A Comparative study on multiobjective reliable and robust optimization for crashworthiness design of vehicle structure, Structural and Multidisciplinary Optimization (2013) 1-16.

[38] M. McKay, R. Beckman, W. Conover, A comparison of three methods for selecting values of input variables in the analysis of output from a computer code, Technometrics 21 (2) (1979) 239-245.

[39] J. H. Friedman, Multivariate adaptive regression splines, Annals of Statistics 19 (1991) 1-141.

[40] J. Friedman, C. Roosen, An introduction to multivariate adaptive regression splines., Statistical methods in medical research 4 (3) (1995) 197-217.

[41] J. E. Eddy, K. Lewis, Effective Generation of Pareto Sets using Genetic Programming, Proceedings of ASME Design Engineering Technical Conference .

[42] G. R. Cook, W. H. Johson, A constitutive model and data for metals subjected to large strains, high strain rates and high temperatures, Proceedings of Seventh International Symposium on Ballistics .

[43] M. Costas, J. Díaz, L. Romera, Hernández, A multi-objective surrogate-based optimization of the crashworthiness of a hybrid impact absorber, International Journal of Mechanical Sciences. Under review .

[44] BASF, Ultramid (R) A3WG10 BK00564 Polyamide 66. Product information sheet, 2012.

[45] M. Huang, Vehicle Crash Mechanics, CRC Press, 2002. 
[46] SAE Standard J211: Instrumentation for Impact Test, Tech. Rep., Safety Test Instrumentation Stds Comm, 1995.

[47] European New Car Assessment Programme (Euro NCAP), Frontal Impact Testing Protocol, Version 6.0.2, 2013.

[48] ABAQUS 6.13 Documentation, Dassault Systèmes, 2013.

[49] B. Adams, L. Bauman, W. Bohnhoff, K. Dalbey, M. Ebeida, J. Eddy, M. Eldred, P. Hough, K. Hu, J. Jakeman, L. Swiler, D. Vigil, DAKOTA, A Multilevel Parallel Object-Oriented Framework for Design Optimization, Parameter Estimation, Uncertainty Quantification, and Sensitivity Analysis: Version 5.3.1 User's Manual, Sandia National Laboratories, December 2009. Updated April 2013. 